\title{
Almanah 2013: novel non-coronary cardiac interventions
}

The national society journals present selected research that has driven recent advances in clinical cardiology. The article was first published in Heart 2013;99:18 1309-1316 Published Online First: 30 July 2013 doi:10.1136/heartjnl-2013-304430, and is republished with permission.

\section{Pascal Meier ${ }^{1,2}$, Olaf Franzen ${ }^{3}$, Alexandra J Lansky ${ }^{2}$}

${ }^{1}$ Yale-UCL Cardiovascular Research Program, The Heart Hospital, University College London Hospitals UCLH, London, UK, ${ }^{2}$ Division of Cardiology, Yale Medical School, New Haven, Connecticut, USA, ${ }^{3}$ Division of Cardiology, Rigshopitalet University Hospital Copenhagen, Copenhagen, Denmark

Abstract

Recent innovations in interventional cardiology have dramatically expanded the therapeutic options for patients with cardiac conditions. Interventional cardiology is no longer limited to the treatment of coronary artery disease but allows also treatment of valvular disease, stroke prevention, hypertension, etc. One of the most important new treatment options is the percutaneous treatment for aortic valve stenosis (transcatheter aortic valve implantation), since aortic valve disease is a rather common problem in elderly patients, with many of them at high risk for surgery. Similarly, mitral regurgitation is often associated with comorbidities which make surgery high risk. The MitraClip is a promising percutaneous alternative to surgical valve repair or replacement. Other procedures discussed in this review are the percutaneous left atrial appendage closure as a non-pharmacologic therapy to prevent strokes, and renal denervation for resistant hypertension. This review explains the basic principles of these procedures, the most important clinical evidence, and also provides additional recent clinical data on each of these them.

\section{Introduction}

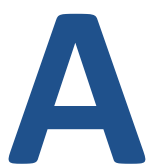

fter Andreas Gruentzig's pioneering balloon angioplasty, percutaneous coronary interventions became the mainstay of cardiology for the ensuing decades ${ }^{1}$; that is until very recently when cardiology has adopted innovations which can be regarded as revolutionary as Gruentzig's angioplasty. Foremost in the development of percutaneous treatment options for aortic valve stenosis, transcatheter aortic valve implantation (TAVI) has improved the treatment options for elderly patients with aortic valve stenosis. Other important developments are the percutaneous treatment options for mitral regurgitation (MR) (MitraClip), non-pharmacologic therapy to prevent cerebral embolisation in patients with atrial fibrillation (AF) such as left atrial appendage (LAA) closure and closure of the patent foramen ovale, and renal denervation to treat resistant hypertension.

\section{Left Atrial Appendage Closure}

$A F$ is very prevalent and the main cause of stroke. The lifetime risk of developing AF is approximately 1 in $4 .{ }^{2}$ It is likely that the true prevalence is underestimated, because it can be difficult to detect paroxysmal AF. Patients with paroxysmal AF probably have a risk of stroke that is similar to patients with persistent $A F^{3}$
Oral anticoagulation has always been the first line treatment to prevent stroke, but it comes with considerable risks, The narrow therapeutic window of warfarin forces a delicate balance between lack of efficacy and a significantly elevated risk of bleeding, therefore requiring frequent blood tests. Additionally, numerous food and drug interactions exist which have a major impact on the patient's daily life. Up to $40 \%$ of patients with AF have contraindications to anticoagulation therapy. Even in trial settings, a relevant proportion of patients are either subor supratherapeutic on warfarin. In a study of 41900 patients with chronic $A F$, only $70 \%$ of patients treated with warfarin remained on this therapy at 1 year, further highlighting difficulties with anticoagulation.

Among patients with non-valvular $A F$, the vast majority of thrombi evolve from the LAA. The fibrillating LAA is a cul-de-sac that creates a milieu for blood stasis and thrombus formation. Therefore, one could expect that exclusion of the LAA from the circulation could reduce the risk for stroke. Several methods have been developed-surgical ligation or amputation and percutaneous catheter based occlusion with specific occlude devices.

Surgical ligation or amputation has been used for many years even though there is very little evidence regarding its effectiveness. ${ }^{5}$ Of course, it is only performed as a 
'bystander' operation in the case of, for example, valve surgery, not as a stand alone procedure. ${ }^{6}$

Percutaneous methods have been developed since 2002. Preliminary studies of two systems specific- ally designed for this purpose (Percutaneous LAA Transcatheter Occlusion (PLAATO) and Watchman systems) have been completed. ${ }^{7}$ These devices are deployed via a venous access and transseptal crossing into the left atrium (LA). These devices are CE (European Conformity) marked in Europe but are not approved by the US Food and Drug Administration (FDA) for clinical use yet.

In addition to these two systems, the Amplatzer cardiac plug and the Lariat (snare device) system are also available.

\section{PLAATO system}

The PLAATO system was a device that was placed in the LAA via a transseptal catheter. It had a self- expanding nitinol frame that was covered by a fabric that was impermeable to blood, thus sealing the LAA and preventing thrombus formation or dislodgement. However, there were adverse effects during follow-up-for example, pericardial effusion in eight patients, two strokes, two transient ischaemic attacks, and three nonprocedural deaths. ${ }^{8}$ The manufacturer has discontinued development of the PLAATO device.

\section{Watchman device}

The Watchman device also involves an expandable device deployed in the LAA via a transseptal catheter. The implanted device has a self-expanding nitinol frame to secure it in the LAA. Unlike the PLAATO device, the fabric of the Watchman device is permeable to blood. ${ }^{9}$ For this reason, patients require conventional thromboembolic prophylaxis with warfarin until the device is endothelialised (eg, at least 45 days post-implant), at which time transoesophageal echocardiography is performed to ensure endothelialisation. In addition, all patients are treated with both aspirin (81-325 mg) and clopidogrel (75 mg) daily for 6 months.

The Watchman device was evaluated in the PROTECT AF (Watchman Left Atrial Appendage System for Embolic Protection in Patients with AF) trial in which over 700 patients with non-valvular AF were randomly assigned in a $2: 1$ ratio to either the device (with the above antithrombotic regimen) or to long term warfarin (international normalised ratio 2.0 to 3.0$).{ }^{10} \mathrm{It}$ had a non-inferiority design. Inclusion criteria allowed for patients with paroxysmal, persistent, or permanent $A F$ and all patients had a CHADS2 score $\geq 1$.

The trial confirmed non-inferiority of Watchman atrial appendage occlusion compared to warfarin therapy regarding the primary end point, a composite of stroke, systemic embolism, and cardiovascular death with a risk ratio of 0.62 (95\% $\mathrm{Cl} 0.35$ to 1.25$).{ }^{11}$ However, the primary safety end point (composite of major bleeding, pericardial effusion, procedure related stroke, and device embolisation) was increased in the device group (7.4 vs 4.4 events per 100 patient-years, respectively). Most of the events in the device group occurred early.
Of these, about $50 \%$ were pericardial effusions requiring drainage.

Two later registries showed improving safety of this device, probably due to a learning curve effect. The rate of complications within 7 days were $3.7 \%$ as compared to $7.7 \%$ in the initial randomised trial. ${ }^{1112}$

\section{Amplatzer septal occluder and Amplatzer cardiac plug}

The Amplatzer septal occluder uses a simpler technique than the PLAATO technique. Instead of general anaesthesia as used in the PLAATO procedure, it is often implanted under local anaesthesia. A case series involving 16 patients demonstrated the use of the Amplatzer septal occluder to close LAA under local anaesthesia without echocardiographic guidance. ${ }^{13}$ The Amplatzer cardiac plug is another device designed specifically for closure of the LAA and is undergoing clinical trials. ${ }^{14} \mathrm{Th}$ is device is nitinol based and consists of a left atrial disk and a distal plug connected to the left atrial disk by a short waist. The distal plug contains six pairs of barbs designed to increase stability within the appendage. This device is shorter than the Watchman device and may be more advantageous in individuals with the variable morphology of the appendage. Animal data have been published demonstrating uncomplicated device delivery with complete occlusion of the appendage at 30-day and 90-day follow-ups. ${ }^{1415}$

The future role of this procedure will also depend on other alternatives to warfarin therapy, such as the novel anticoagulants. So far, there is no strong evidence suggesting they are superior to warfarin, apart from rivaroxaban which showed a lower bleeding risk, but they are a promising alternative to warfarin and much easier to use. ${ }^{16}$

The 2012 focused update of the European Society of Cardiology (ESC) guidelines for the management of AF makes a weak recommendation for the use of interventional, percutaneous LAA closure in patients with a high stroke risk and contra- indications for long term anticoagulation. ${ }^{17}$ In conclusion, percutaneous LAA closure seems as effective as warfarin according to one randomised trial, but comes with periprocedural complications (such as pericardial effusion).

Left atrial appendage closure:
- Percutaneous LAA closure is a promising alternative
to war farin therapy in patients with AF who have a
high stroke risk.
- Data are scarce and the procedure should be lim-
ited to patients who have clear contraindications for
warfarin.
- Novel anticoagulants (eg, rivaroxaban) represent
another alternative for patients with a contraindica-
tion for warfarin.

\section{Mitral Valve Interventions}

The prevalence of moderate or severe MR is over $10 \%$ in those older than 75 years and the natural course is often fatal. ${ }^{18}$ However, patients with chronic severe MR 
often have other comorbidities that increase their risk for cardiac surgery. There is an urgent need for a less invasive percutaneous approach. Several approaches have been tested. Currently, the most promising one is the MitraClip system (Abbott Laboratories, Abbott Park, Illinois, USA). It is based on the surgical Alfieri stitch technique, an edge-to-edge repair. ${ }^{19}$

The MitraClip system consists of a catheter to guide the path of the clip delivery and a clip delivery system which includes the detachable clip with a Dacron cover to enable tissue ingrowth. The clip delivery system has a control mechanism to open and close the two arms of the clip. Tissue of the mitral leaflet is held between the arms and each side of the $U$-shaped gripper. Then, the clip is closed and locked so that the two leaflets stay approximated for repair.

Usually under general anaesthesia, the guide catheter is inserted through the femoral vein to reach the left atrium through a transseptal approach. Using this path, the MitraClip is delivered and deployed. The clip delivery system aligns the MitraClip with the line of coaptation, tissues of the mitral valve leaflet are grasped, and the clip is partially closed to about $60^{\circ}$. Ideal length for coaptation is at least $2 \mathrm{~mm}$. Transoesophageal echocardiography (TOE) is used to guide the deployment of the clip and later to evaluate the reduction in MR. Periprocedural imaging is key for this procedure, as it should be the case for surgical mitral valve repair; the mitral valve is a relatively complex structure..$^{20}$ If there is adequate leaflet insertion at $60^{\circ}$ of clip closure, the clip is further closed to appose the leaflets and induce coaptation to reduce $M R$ maximally in patients with either degenerative or functional MR. In order to avoid a stenosis following placement of the MitraClip, the baseline mitral valve area should have been $>4 \mathrm{~cm}^{2}$.

The initial human clinical experience with MitraClip was studied in the EVEREST (Endovascular Valve Edge-toEdge Repair Study) phase I study. ${ }^{21}$ The study included 27 patients. Fourteen of the patients had MR reduced to $\leq 2+$ at 6 months. The study showed promise for MR patients at high risk for con- ventional surgical procedures.

The pivotal EVEREST II trial randomised 279 patients with chronic moderately severe or severe MR (grade 3+ or $4+$ ) to undergo either percutaneous MitraClip or mitral valve surgery in a 2:1 ratio. ${ }^{22}$ Although before hospital discharge percutaneous therapy was less effective at reducing $M R$, the rates of MR reduction at 12 and 24 months were similar in both the groups. However, percutaneous therapy was found to be safer with a reduced rate of major adverse events at 30 days. The study showed sustained clinical improvement, as assessed by quality of life, heart failure status, and left ventricular function.

The EVEREST II study was not specifically focused on very high risk patients. The mean age was 66 years, mean ejection fraction was $60 \%$, and major comorbidities were rare. In the EVEREST II High Risk Study (HRS), however, patients had severe MR (3-4+), a mean age of 77 years, and an estimated surgical mortality rate of $\geq 12 \%$. ${ }^{23}$ More than $50 \%$ of these patients had under- gone previous cardiac surgery. They were compared retrospectively with a group of patients who were screened con-currently but not enrolled into the EVEREST II study. These patients had been treated by standard medical therapy.

The 30-day procedure related mortality rate was similar in both the groups $(7.7 \%$ in the HRS vs $8.3 \%$ in the comparator group). The 12-month survival was higher in the HRS group (76\% vs 55\%). In surviving patients the baseline and 12-month data were compared. MR grade had reduced to $\leq 2+$ in $78 \%$ patients. There was improvement in left ventricular end-diastolic volume (172-140 $\mathrm{mL})$, end-systolic volume $(82-73 \mathrm{~mL})$, New York Heart Association functional class (III/IV at baseline in $89 \%$ to class I/II in 74\%; $p<0.0001$ ), quality of life and mental component score at 12 months.

Feldman et $\left.a\right|^{24}$ reported clinical results of use of the clip in a cohort of the first 107 patients followed for as long as 3 years. These were 55 patients treated in the EVEREST phase I feasibility trial, and 52 roll-in patients treated in the EVEREST II pivotal trial representing the prerandomisation start-up experience. Out of the successfully treated patients, $66 \%$ achieved the primary end point of freedom from death, mitral valve surgery, or $\mathrm{MR}>2+$ at 12 months. There was sustained freedom from death, surgery or recurrent MR in most patients even at 3 years.

The MitraClip device has received a CE approval and has already been used in thousands of patients worldwide. ${ }^{25}$ ${ }^{26}$ Until approval is obtained from the FDA, the clip is available only through the REALISM continued access registry in the USA. Patients enrolled into this 'realworld' registry are assigned to either the high risk arm or the non-high risk arm. They are followed up at 30 days, 6 months, and 12 months.

Interim results of the EVEREST II REALISM study were presented at the Society for Cardiovascular Angiography and Interventions (SCAI) 2011 scientific sessions. ${ }^{27-29}$ On average, the age of patients in the registry was over 70 years. The MitraClip procedure was safe with a 30-day mortality of $3.8 \% .{ }^{28}$ At 1 year, $83 \%$ of patients in the high risk group had only mild to moderate MR (1+ or $2+$ ). Before the procedure, the quality of life scores were worse for functional MR than for degenerative $M R,{ }^{29}$ but there was also greater improvement after the procedure.

An analysis of the first 100 patients in the MitraSwiss register from Switzerland showed that congestive heart failure before clip implantation and previous coronary artery bypass grafting (CABG) were predictors of poor outcome with the MitraClip procedure. ${ }^{26} \mathrm{~A}$ reduction of $M R$ to $\leq 2+$ during the procedure and a low grade MR at discharge predicted better mid-term survival. Gaemperli et $\mathrm{al}^{30}$ documented an improvement in the haemodynamic profile immediately after the procedure. This acute haemodynamic improvement was associated with favourable mid-term outcome.

In conclusion, the MitraClip implantation is inferior to surgical mitral repair for reducing MR grade, but it is probably safer in higher risk patients. Even though the reduction in $\mathrm{MR}$ is less than with surgery, the subjective symptom im- 
provement is comparable. The most suitable patients are those at high risk for surgery and particularly those with functional MR. The optimal time point for the procedure is not clear, but for patients undergoing surgery the data indicate that sooner is probably better. ${ }^{31}$ This makes sense, as surgery has become safer and has changed the riskbenefit considerations. Since the percutaneous approach has further reduced the periprocedural risk, the same consideration should apply here as well.

Mitral valve interventions:

Key points

- Data for the MitraClip system procedure are very limited and derive mainly from the EVEREST II trial.

- Even though the EVEREST trial did not enrol very high risk patients, the procedure should be limited to those at high surgical risk because of the limited effect.

\section{Aortic Valve Interventions}

Aortic valve stenosis (AS) is a very common disease, with increasing prevalence at older age. ${ }^{32}{ }^{33}$ In properly selected patients, aortic valve replacement (AVR) substantially improves symptoms and increases life expectancy. However, for patients with major comorbidities, as is often the case in this elderly patient group, AVR surgery may not be appropriate because of the risks involved. A less invasive means is percutaneous aortic valvuloplasty, but this has a limited and usually only a temporary effect. Transcatheter aortic valve replacement (TAVR or TAVI) provides an alternative for treating AS in patients at high surgical risk. It is also a preferred option in patients who may have technical issues with surgery-for example, porcelain aorta or prior mediastinal radiation, or surgery with dense adhesions, or prior sternal infection with complex reconstruction, or a patent left internal mammary graft.

The most recent 2012 European guidelines state that TAVR is recommended in patients with severe symptomatic AS who are, according to the local 'heart team' (multidisciplinary team), considered unsuitable for conventional surgery because of severe comorbidities. Among high risk patients who are potential candidates for surgery, the decision should be individualised and discussed in a 'heart team'. Risk scores can be helpful for the clinical decision making. The logistic EuroSCORE $\geq 20 \%$ is very established and often used in this setting as well; a score $\geq 20 \%$ has been proposed to define high risk. However, it generally overestimates operative mortality. Factors such as pulmonary hypertension or right ventricular dysfunction are not accounted for. The newer EuroScore II is probably more useful; alternatively, the Society of Thoracic Surgeons (STS) score can be used with a score $\geq 10 \%$ indicating high risk (a threshold of $\geq 8 \%$ was used in the PARTNER A trial ). We have to be aware that no 'perfect' TAVI risk score has been established yet; they have been developed for patients undergoing cardiac surgery, pre- dominantly CABG. These scores do not consider factors which are important when debating TAVR versus surgical AVR, such as frailty, porcelain aorta, history of chest radiation or patent coronary bypass grafts. Therefore, the final decision should be based on a comprehensive clinical judgement. Importantly, TAVI should currently not be performed in patients at intermediate surgical risk until more data for this group of patients are available, for example, from the ongoing SURTAVI (Surgery and Transcatheter Aortic Valve Implantation) trial (NCT01586910). ${ }^{34}$

The 2012 American College of Cardiology (ACC) guidelines are very similar and equally endorse a multidisciplinary team decision for patients at high risk for surgery, defining 'too high risk for surgery' as an estimated $\geq 50 \%$ risk of death or irreversible morbidity at 30 days. ${ }^{35}$ Even though these guidelines are very recent, with increasing experience the indication for TAVR is rapidly expanding in some countries. In selected centres in Germany, TAVR accounts for over one-third of all AVR procedures. TAVR is a cost effective treatment for those who are not eligible for surgical AVR. ${ }^{36}$ In the USA, the introduction of TAVR has been somewhat slower, mainly due to regulatory reasons. ${ }^{35}{ }^{37}$ Interestingly, in most centres, the introduction of TAVI has been associated with increase in conventional surgical AVR activity also. ${ }^{38} 39$ Currently, two valves are in widespread use and have FDA approval-the balloon expanding Edwards Sapien transcatheter valve (THV) (Edwards Lifesciences, Irvine, California, USA) and the self-expanding CoreValve (Medtronic Inc, Minneapolis, Minnesota, USA).

The landmark trials for TAVR, the PARTNER (Placement of Aortic Transcatheter Valve) trials, have been performed with the Edwards Sapien valve. The subsequent Sapien Aortic Bioprosthesis European Outcome (SOURCE) Registry assessed results of the use of the Edwards Sapien valve in consecutive patients in Europe, with procedural success rates as high as $93.8 \%$ and a low incidence of procedure related complications ${ }^{40}$ Incidence of stroke was similar (2.5\%) with both the transfe moral and the transapical approaches. The 30 -day mortality was lower $(6.3 \%$ vs $10.3 \%)$ and 1 -year survival was higher $(81.1 \%$ vs $72.1 \%$ ) in patients with the transfemoral approach. 4041 However, there was a much higher rate of vascular complications with transfemoral TAVR ( $22.9 \%$ vs $4.7 \%)$, probably because of the larger diameter of the delivery sheath.

Most TAVR teams prefer the transfemoral approach as it avoids surgical manipulation of the chest and reduces post- operative pain. ${ }^{42}$ It is also the least invasive method. ${ }^{43}$ However, thoracic epidural analgesia provided during transapical TAVR can significantly reduce pain and periprocedural respiratory complications. ${ }^{44}$

The Edwards Sapien XT valve has a cobalt chromium frame with struts that are thinner and have a more open structure.$^{45} \mathrm{~A}$ trial on 120 patients showed that it had the same short term performance as the earlier SAPIEN valve but was associated with threefold lower risk of major vascular complications. ${ }^{46}$

The PARTNER valve trial was the world's first prospective randomised controlled trial for TAVR. It was designed with two arms:

- PARTNER A randomised 699 high surgical risk patients to either TAVR or surgical AVR

- PARTNER B randomised 358 inoperable patients to either TAVR or standard medical care. 
The 30-day mortality was higher in TAVR patients than in those administered standard medical care ( $5 \%$ vs $2.8 \%, p=0.41$ ) but was less for TAVR than in those undergoing open surgical AVR (3.4\% vs $6.5 \%, p=0.07) .{ }^{47}$

In the PARTNER A cohort, those who underwent TAVR had a higher incidence of major strokes (3.8\% vs $2.1 \%$ at 30 days; $5.1 \%$ vs $2.4 \%$ at 1 year) and major vascular complications ( $11.0 \%$ vs $3.2 \%$ at 30 days; $11.3 \%$ vs $3.5 \%$ at 1 year). Those treated with surgical AVR had a higher incidence of major bleeding (19.5\% vs $9.3 \%$ at 30 days; $25.7 \%$ vs $14.7 \%$ at 1 year) and new AF (16.0\% vs $8.6 \%$ at 30 days; $17.1 \%$ vs $12.1 \%$ at 1 year).

Both surgical AVR and TAVR resulted in a decrease in aortic valve gradients and an increase in effective orifice area (EOA) ( $p<0.0001)$ which remained stable over 2 years. TAVR was associated with higher indexed EOA, lower prosthesis-patient mismatch, and more aortic regurgitation $(A R) .{ }^{48}$

Though right ventricular function is reduced following surgical AVR, there is no such effect with TAVR. ${ }^{49} 50$

$A$ recent study has shown that there is transient systolic and diastolic dysfunction within the first $24 \mathrm{~h}$ of a successful TAVR. ${ }^{51}$ This is associated with an increase in serum markers of myocardial injury and dysfunction suggesting that the post- procedural dysfunction is due to myocardial stunning and periprocedural injury to the myocardium.

Post-procedure paravalvular AR is more common after TAVR than after surgery. ${ }^{5253}$ We have learned from the PARTNER trials data, confirmed by subsequent studies, that there is an association of post-TAVR paravalvular leak and increased mortality. Based on the German TAVR registry, the occurrence of significant angiographically assessed AR immediately after TAVR was $17.2 \%$. This population consisted of $84 \%$ Medtronic CoreValve systems and $16 \%$ Edwards Sapien valves. The risk for in-hospital mortality was increased around 2.5-fold in patients with significant AR..$^{52}$ However, whether AR is a cause for mortality or just a marker for higher risk patients (severe calcification, tighter valves) is unclear. In this study, AR was an independent predictor of mortality in an adjusted analysis, but of course such adjustments are rarely able to eliminate con-founding entirely.

The survival in both the PARTNER A and PARTNER B trials was remarkably good. But stroke and perivalvular leakage associated with the Sapien valve required further evolution of the device.

Two large studies are being conducted with the Sapien XT device: the PARTNER II study and the ARTE (Aspirin Versus Aspirin and Clopidogrel Following Transcatheter Aortic Valve Implantation) trial. The later study is comparing the efficacy of aspirin versus a combination of aspirin and clopidogrel following TAVR in preventing major ischaemic events. ${ }^{45}$

The PARTNER II study has recently started.$^{45}$ It consists of two arms: cohorts A and B. Cohort A will have 2000 patients with an STS risk score of $\geq 4$. They will be randomised on a $1: 1$ basis to TAVR with the Edwards Sapien $X T$ valve or to surgical AVR. There will be substratification based on coronary artery disease (CAD). Patients with CAD will be randomised on a $1: 1$ basis to TAVR plus per- cutaneous coronary intervention and to surgical AVR plus CABG. A detailed pre- and post-procedural logical assessment will be done on all patients. Data for a frailty substudy will also be collected. Cohort B will have 500 inoperable patients who will be randomised on a $1: 1$ basis to TAVR with the Edwards Sapien THV and Edwards Sapien XT devices. The safety and efficacy of the two devices will be compared. The study is expected to finish in 2018.

Edwards Lifesciences (USA) has developed two additional valves: the Centera and the Sapien III valves. Both these devices have recently entered first-in-man studies.

Other than the Sapien device, the only other FDA approved device is the CoreValve (Medtronic Inc, Minneapolis, Minnesota, USA). It has porcine pericardial leaflets mounted on a self-expanding nitinol frame. It does not allow antegrade implantation, unlike the Sapien valve which can be implanted both antegrade as well as retrograde. ${ }^{54}$ However, the advantage is that it uses a lower profile delivery system of 18 French.

In the Medtronic CoreValve multicentre expanded evaluation registry, the initial procedural success was high $(97 \%)$ and the procedural mortality was low $(1.5 \%) .{ }^{55}$ The 30-day all cause mortality (including procedural) was also low (8\%). These benefits were sustained over time up to 1 year. ${ }^{56}$ Ussia et al reported sustained clinical and functional cardiovascular benefits over 3 years too. A recent presentation of the results of the ADVANCE CoreValve registry revealed an all cause mortality rate of $12.8 \%$ and a cardiac mortality rate of $8.4 \%$ at 6 months. Stroke rates were low (2.9\% at 30 days) while the pacemaker implantation rate was $26.3 \%{ }^{45}$

This rather high rate of pacemaker implantation is often regarded as a limitation for the CoreValve system, when com- paring it with the rates for the Edwards Sapien system and with the rate after surgical AVR. After surgical $A V R$, a recent large cohort study in 780 patients showed a need for a pacemaker implantation post-procedure of $3.2 \% .{ }^{57}$ However, the need for pacemaker for the selfexpandable CoreValve system has decreased over time with technical iterations and operator's experience. The valves are now implanted in a higher position, which has significantly reduced electrical disturbances.

MRI tests have shown multiple small cerebral infarcts (in 77\% cases) following TAVR. Most of the lesions were silent. Clinical stroke was associated with higher infarct number and volume. ${ }^{58}$ The SIMPLIFy TAVI study (Transcatheter Aortic Valve Implantation Without Predilation) is investigating whether the avoidance of balloon valvuloplasty for predilation of the native aortic valve reduces the stroke risk during TAVI.

The CoreValve Advance II prospective registry is expected to define ways to reduce the need for permanent pacemaker implantation.

TAVR has also been tried successfully in several 'off label' indica- tions such as in patients with bicuspid valves, severe MR, reduced left ventricular ejection fraction, and low gradient, low output AS. ${ }^{59}$

Other valves are currently under evolution. These include the Sadra Lotus valve (Medtronic Inc, Minneapolis, Minnesota, USA), Direct Flow Medical (DFM) valve (Direct Flow Medical Inc, Santa Rosa, California, USA), 
Symetis Acurate valve (Symetis SA, Ecublens, Switzerland), JenaValve ( JenaValve, Munich, Germany), and the Engager valve.

\section{Embolic protection devices}

Cerebrovascular adverse events have been reported as complications of TAVI. Also, MRI studies have detected subclinical post- procedural embolic lesions following TAVR in over $90 \%$ of patients. ${ }^{60}$ To address this issue, specific embolic protection devices are being developed. The Claret CE Pro system (Claret Medical, Inc, Santa Rosa, California, USA) has two filters to capture any debris that moves in the brachiocephalic and left common carotid artery. Naber et a ${ }^{61}$ described the firstin-man use of this novel device in 40 patients undergoing TAVI with evidence of reduction of procedural cerebral embolic burden.

The TriGuard Cerebral Protection Device (Keystone Heart, Israel, formerly SMT R\&D) works as a debris deflector instead of a debris collector. A similar device is the Embrella Embolic Deflector (Edwards Lifesciences, Irvine, California, USA). Both Embrella and TriGuard have a protective shield that deflects embolisms away from the cerebral arteries.

The SMT FIM ( first in man) feasibility study described the use of the SHEF device in the first 15 patients. ${ }^{62}$ The ongoing DEFLECT I (SMT Embolic Deflection CE Mark) study is expected to provide further evidence of the effectiveness of the device. First results have been presented at EuroPCR in May 2013 in Paris and show promising results. The maximum total lesion volume in DEFLECT I was $95 \%$ smaller than the maximum total lesion volume in reported studies $\left(3.94 \mathrm{vs} 70.3 \mathrm{~cm}^{3}\right)$.

The human experience with Embrella comes from a small study of four patients with severe AS who underwent aortic balloon valvuloplasty or TAVI. ${ }^{63}$ There were no procedural complications. The procedural safety, technical feasibility, and exploratory efficacy of the Embrella device was studied in the ProTAVI-C pilot study at nine centres involving 54 patients. Use of the Embrella deflector system during TAVI was feasible and safe with minimal procedural complications. There were no procedural strokes or impairment of neurocognitive function. Though there was cerebral microembolisation in all patients, there was a potential decrease in cerebral lesion volume. A phase 2 randomised study is being conducted to measure the reduction of new cerebral lesion volume.

Aortic valve interventions:

Key points

- There is strong evidence demonstrating that TAVR is effective compared to medical therapy.

- TAVR is non-inferior to surgical AVR in high risk patients, it has several advantages, but has been associated with a slightly higher stroke risk.

- Ongoing and planned trials looking at the comparative effect of TAVR and AVR in intermediate risk patients are likely to show non-inferiority and may further increase the use of TAVR.

\section{Renal Denervation}

Approximately $5-10 \%$ of all hypertensive patients are resistant to medical treatment, defined as blood pressures $>140 / 90$ mm Hg or $>130-139 / 80-85$ mm Hg in diabetic patients, or $>130 / 80 \mathrm{~mm}$ Hg in patients with chronic kidney disease (CKD) in the presence of $\geq 3$ antihypertensives of different classes, including a diuretic, at maximal or the highest tolerated dose. However, 'resistance' is probably not rarely caused by malcompliance. On the other hand, drug intolerance or side effects are not rare and can be a challenge in medical blood pressure management. The development of catheter based radiofrequency ablation of the renal sympathetic nervous fibres is a highly promising new approach.

In 2009, a European-Australian proof-of-principle study, the non-randomised Simplicity HTN-1, was published; it was per-formed on 50 patients with resistant hypertension (ie, systolic blood pressure $\geq 160 \mathrm{~mm} \mathrm{Hg}$ on three or more antihypertensive medications, including a diuretic).$^{64}$ Five of the patients who were not anatomically eligible for the denervation procedure were used as controls. After this radiofrequency ablation catheter procedure (Symplicity, Ardian Inc, Palo Alto, California, USA), the blood pressure in these 45 patients dropped significantly over a period of 12 months.

An expanded cohort of 153 patients with resistant hypertension was studied in the Simplicity HTN-1 study. ${ }^{65}$ The patients were treated with catheter based renal sympathetic denervation at 19 centres in Australia, Europe, and the USA. They showed a substantial and sustained reduction of blood pressure over a follow up of $\geq 2$ years without any significant adverse effects. Simplicity HTN-1 was a feasibility study and had no control group. The subsequent Simplicity HTN-2 trial randomised 106 patients to renal denervation or control. ${ }^{66}$ At 6 months, the blood pressure in the treatment group was significantly lower. After the 6-month end point, a crossover was done and renal denervation in control patients was allowed. ${ }^{67}$ In the initial renal denervation group, the significant reduction in mean blood pressure at 6 months $(-32 \mathrm{~mm} \mathrm{Hg})$ was sustained at 12 months $(-28 \mathrm{~mm} \mathrm{Hg})$. At 6 months, the control group had shown an increase in blood pressure from $182.8 \pm 16.3$ to $190.0 \pm 19.6 \mathrm{~mm} \mathrm{Hg}$. Those among this control group who underwent renal denervation as crossover had a significant fall in their blood pressure at 6 months after the procedure $(-24 \mathrm{~mm} \mathrm{Hg} ; \mathrm{p}<0.001$ for difference from before the procedure). This sub- stantiated the safety and efficacy of renal denervation via controlled radiofrequency ablation.

The Simplicity HTN-2 study did not report on the $24 \mathrm{~h}$ blood pressure monitoring. It was not a blinded study design. These methodological issues are being addressed in the Simplicity HTN-3 study. ${ }^{67}$ It is a multicentre, single blind, randomised controlled trial where patients are being randomised to bilateral renal denervation with the Simplicity catheter or to a sham procedure. One of the important secondary end points of the study is the change in $24 \mathrm{~h}$ blood pressure. The Simplicity renal denervation device has also been tried in other patient groups, such as in a pilot study of 
patients with resistant hypertension and moderate to severe (stage 3 or 4) CKD. ${ }^{68}$ It was found to be safe and effective. The Simplicity HF study is currently enrolling 40 patients for a pilot study to evaluate the effects of renal denervation in heart failure patients. In the real world setting, the Global Simplicity Registry is expected to enrol 5000 patients in 200 sites worldwide. The registry had enrolled 617 patients by 25 May 2013. Based on preliminary data presented at the EuroPCR 2013, there have been significant drops in in-office blood pressure and $24 \mathrm{~h}$ blood pressure. These reductions are, however, smaller than those seen in clinical trials.

At the EuroPCR 2013, interim data from the first 41 patients treated with the alternative Vessix renal denervation system in its REDUCE-HTN study were presented. ${ }^{69}$ At 6 months, there was a significant reduction in blood pressure $(-27.6 \mathrm{~mm} \mathrm{Hg} ; \mathrm{p}<0.0001)$. In those patients for whom 12-month data were available, there was a sustained reduction in systolic blood pressure $(-28.4 \mathrm{~mm}$ $\mathrm{Hg}$ ). There were no device related adverse events or procedural complications and the procedure time was short.

The EnlighHTN multielectrode renal denervation catheter (St Jude Medical) has been tried in 46 patients who were then followed up for 1 year. ${ }^{70}$ Most patients (80\%) responded to therapy (had at least $10 \mathrm{~mm} \mathrm{Hg}$ reduction in mean blood pressure). The mean reduction in blood pressure at 12 months was $27 \mathrm{~mm} \mathrm{Hg}$. The multielectrode device reduces the renal denervation time and is also less painful to patients. Other devices with a multimode approach include the Covidien One-Shot device and a modification of the Simplicity device called the Spyral device.

Currently, renal denervation is considered an adjunctive therapy to medical treatment, not a replacement, since the average number of patients taking antihypertensive medications has not declined in the trials in spite of the reduction in mean blood pressure following renal denervation. ${ }^{70}$

Although there is a lot of excitement about this new modality of treatment for resistant hypertension, there are concerns about the diffuse renal artery constriction and tissue damage at the ablation site, with oedema and thrombus formation that may occur following renal nerve ablation. ${ }^{71}$ Dual antiplatelet therapy may therefore be needed during the procedure. Even though renal denervation has been tried in patients with CKD, ${ }^{68}$ patients with high grades of renal insufficiency should only be treated and systematically followed in clinical trials. It should also not be tried in anatomically unsuitable renal arteries (diameter $<4 \mathrm{~mm}$; length $<20 \mathrm{~mm}$; fibromuscular dysplasia; significant renal artery stenosis) or in cases of secondary and treatable causes of hypertension. ${ }^{72}$ We also have to be aware that there are no data on the impact of renal denervation on clinical outcomes at this stage. Its use should therefore be restricted to patients with severe, resistant hypertension and it should be regarded as an adjunctive and not an alternative therapy to antihypertensive drugs.
Renal denervation:

Key points

- Renal denervation is a promising approach to treat resistant hypertension, its effect on blood pressure reduction is impressive, but future studies need to prove that this translates into improved clinical outcomes. - Similar to TAVR, it is likely that the indication for this procedure will further expand to non-resistant hypertension and to other areas such as heart failure, rate control for AF, etc.

\section{Conclusion}

While there is clearly significant technological progress in percutaneous coronary interventions,$^{73}$ the expanding options to treat non-coronary cardiac disease with catheter based techniques are revolutionary. Several of these procedures have been developed for very high surgical risk or 'no option' patients, such as TAVR, but are increasingly used in high risk or even intermediate risk patients as a less invasive alternative to surgery. The fast technological progress, increasing understanding, and improvements in the operators' experience will further expand the indications for these procedures to lower risk patients and for applications for other indications. Renal denervation, as an example, may show benefit in patients with heart failure or for rate control in AF.

Contributors PM drafted the manuscript. OF revised the manuscript critically for intellectual content. AJL revised the manuscript critically for intellectual content. All three authors contributed significantly to this paper and have approved the final version.

\section{References}

1 Meier P, Timmis A. Almanac 2012: interventional cardiology. Anadolu Kardiyol Derg 2012;13:91-101.

2 Lloyd-Jones DM, Wang TJ, Leip EP, et al. Lifetime risk for development of atrial fibrillation: the Framingham Heart Study. Circulation 2004;110:1042-6.

3 European Heart Rhythm Association, European Association for Cardio-Thoracic Surgery, Camm AJ, et al. Guidelines for the management of atrial fibrillation: the task force for the management of atrial fibrillation of the European Society of Cardiology (ESC). Eur Heart J 2010;31:2369-429.

4 Gallagher AM, Rietbrock S, Plumb J, et al. Initiation and persistence of warfarin or aspirin in patients with chronic atrial fibrillation in general practice: do the appropriate patients receive stroke prophylaxis? J Thromb Haemost 2008;6:1500-6.

5 Garcia-Fernandez MA, Perez-David E, Quiles J, et al. Role of left atrial appendage obliteration in stroke reduction in patients with mitral valve prosthesis: a transesophageal echocardiographic study. J Am Coll Cardiol 2003;42:1253-8.

6 MEMBERS WC, Bonow RO, Carabello BA, et al. 2008 Focused update incorporated into the ACC/AHA 2006 guidelines for the management of patients with valvular heart disease: a report of the American College of Cardiology/American Heart Association Task Force on Practice Guidelines (Writing Committee to Revise the 1998 Guidelines for the Management of Patients With Valvular Heart Disease): endorsed by the Society of Cardiovascular Anesthesiologists, Society for Cardiovascular Angiography and Interventions, and Society of Thoracic Surgeons. Circulation 2008;118:e523-661.

7 Khattab AA, Meier B. Transcatheter left atrial appendage exclusion, gold or fool's gold? Eur Heart J Suppl 2010;12:E35-40.

8 Sousa JE, Costa MA, Tuzcu EM, et al. New frontiers in interventional cardiology. Circulation 2005;111:671-81. 
9 Munkholm-Larsen S, Cao C, Yan TD, et al. Percutaneous atrial appendage occlusion for stroke prevention in patients with atrial fibrillation: a systematic review. Heart 2012;98:900-7.

10 Holmes DR, Reddy VY, Turi ZG, et al. Percutaneous closure of the left atrial appendage versus warfarin therapy for prevention of stroke in patients with atrial fibrillation: a randomised non-inferiority trial. Lancet 2009;374:534-42.

11 Reddy VY, Doshi SK, Sievert H, et al. Percutaneous left atrial appendage closure for stroke prophylaxis in patients with atrial fibrillation: 2.3-year follow-up of the PROTECT AF (Watchman Left Atrial Appendage System for Embolic Protection in Patients with Atrial Fibrillation) Trial. Circulation 2013;127:720-9.

12 Reddy VY, Holmes D, Doshi SK, et al. Safety of percutaneous left atrial appendage closure: results from the Watchman Left Atrial Appendage System for Embolic Protection in Patients with AF (PROTECT AF) clinical trial and the Continued Access Registry. Circulation 2011;123:417-24.

13 Massumi A, Chelu MG, Nazeri A, et al. Initial experience with a novel percutaneous left atrial appendage exclusion device in patients with atrial fibrillation, increased stroke risk, and contraindications to anticoagulation. Am J Cardiol 2013;111:869-73.

14 Viles-Gonzalez JF, Kar S, Douglas P, et al. The clinical impact of incomplete left atrial appendage closure with the Watchman Device in patients with atrial fibrillation: a PROTECT AF (Percutaneous Closure of the Left Atrial Appendage Versus Warfarin Therapy for Prevention of Stroke in Patients With Atrial Fibrillation) substudy. J Am Coll Cardiol 2012;59:923-9.

15 Bass JL. Transcatheter occlusion of the left atrial appendage-experimental testing of a new Amplatzer device. Catheter Cardiovasc Interv 2010;76:181-5.

16 Fox BD, Kahn SR, Langleben D, et al. Efficacy and safety of novel oral anticoagulants for treatment of acute venous thromboembolism: direct and adjusted indirect meta-analysis of randomised controlled trials. BMJ 2012;345:e7498.

17 Camm AJ, Lip GY, De Caterina R, et al. 2012 focused update of the ESC guidelines for the management of atrial fibrillation: an update of the 2010 ESC guidelines for the management of atrial fibrillation. Developed with the special contribution of the European Heart Rhythm Association. Eur Heart J 2012;33:2719-47.

18 lung B, Vahanian A. Degenerative calcific aortic stenosis: a natural history. Heart 2012;98:iv7-13.

19 Alfieri O, Denti P. Alfieri stitch and its impact on mitral clip. Eur $J$ Cardiothorac Surg 2011;39:807-8.

20 Delgado V, Kapadia S, Marsan NA, et al. Multimodality imaging before, during, and after percutaneous mitral valve repair. Heart 2011;97:1704-14.

21 Feldman T, Wasserman HS, Herrmann HC, et al. Percutaneous mitral valve repair using the edge-to-edge technique: six-month results of the EVEREST phase I clinical trial. J Am Coll Cardiol 2005;46:2134-40.

22 Feldman T, Foster E, Glower DD, et al. Percutaneous repair or surgery for mitral regurgitation. N Engl J Med 2011;364:1395-406.

23 Whitlow PL, Feldman T, Pedersen WR, et al. Acute and 12-month results with catheter-based mitral valve leaflet repair: the EVEREST II (Endovascular Valve Edge-to-Edge Repair) high risk study. J Am Coll Cardiol 2012;59:130-9.

24 Feldman T, Kar S, Rinaldi M, et al. Percutaneous mitral repair with the MitraClip system: safety and midterm durability in the initial EVEREST (Endovascular Valve Edge-to-Edge REpair Study) cohort. J Am Coll Cardiol 2009;54:686-94.

25 Tamburino C, Ussia GP, Maisano F, et al. Percutaneous mitral valve repair with the MitraClip system: acute results from a real world setting. Eur Heart J 2010;31:1382-9.

26 Sürder D, Pedrazzini G, Gaemperli O, et al. Predictors for efficacy of percutaneous mitral valve repair using the MitraClip system: the results of the MitraSwiss registry. Heart 2013;99:1034-40.

27 Kar S, Rinaldi M, Lim DS, et al. EVEREST II REALISM: a continued access study to evaluate the safety and effectiveness of the MitraClip device: demographics and procedural outcomes. Society for Cardiovascular Angiography and Interventions (SCAI) 2011 Scientific Sessions; Baltimore, 2011.

28 Rinaldi MJ, Kar S, Lim DS, et al. EVEREST II REALISM: a continued access study to evaluate the safety and effectiveness of the Mi-
traClip device: analysis of a 6 month patient cohort. Society for Cardiovascular Angiography and Interventions (SCAI) 2011 Scientific Sessions; Baltimore, 2011.

29 Sarkar K, Ussia GP, Cammalleri V, et al. Quality of life of high risk patients following percutaneous mitral valve repair with the MitraClip system. Society for Cardiovascular Angiography and Interventions (SCAI) 2011 Scientific Sessions; Baltimore, 2011.

30 Gaemperli O, Moccetti M, Surder D, et al. Acute haemodynamic changes after percutaneous mitral valve repair: relation to midterm outcomes. Heart 2012;98:126-32.

31 Samad Z, Kaul P, Shaw LK, et al. Impact of early surgery on survival of patients with severe mitral regurgitation. Heart 2011;97:221-4.

32 Nkomo VT, Gardin JM, Skelton TN, et al. Burden of valvular heart diseases: a population-based study. Lancet 2006;368:1005-11.

33 Rosenhek R. Almanac 2011: valvular heart disease. The national society journals present selected research that has driven recent advances in clinical cardiology. Heart 2011;97:2007-17.

34 Sinning J-M, Werner N, Nickenig G, et al. Transcatheter aortic valve implantation: the evidence. Heart 2012;98:iv65-72.

35 Agnihotri A. 2012 ACCF/AATS/SCAI/STS expert consensus document on transcatheter aortic valve replacement: executive summary. J Thorac Cardiovasc Surg 2012;144:534-7.

36 Watt M, Mealing S, Eaton J, et al. Cost-effectiveness of transcatheter aortic valve replacement in patients ineligible for conventional aortic valve replacement. Heart 2012;98:370-6.

37 Binder RK, Webb JG. TAVI: from home-made prosthesis to global interventional phenomenon. Heart 2012;98:iv30-6.

38 Grant SW, Devbhandari MP, Grayson AD, et al. What is the impact of providing a transcatheter aortic valve implantation service on conventional aortic valve surgical activity: patient risk factors and outcomes in the first 2 years. Heart 2010;96:1633-7.

39 Malaisrie SC, Tuday E, Lapin B, et al. Transcatheter aortic valve implantation decreases the rate of unoperated aortic stenosis. Eur J Cardiothorac Surg 2011;40:43-8.

40 Thomas M, Schymik G, Walther T, et al. Thirty-day results of the SAPIEN aortic bioprosthesis European outcome (SOURCE) registry: a European registry of transcatheter aortic valve implantation using the Edwards SAPIEN Valve. Circulation 2010;122:62-9.

41 Thomas M, Schymik G, Walther T, et al. One-year outcomes of cohort 1 in the Edwards SAPIEN Aortic Bioprosthesis European Outcome (SOURCE) registry: the European registry of transcatheter aortic valve implantation using the Edwards SAPIEN valve. Circulation 2011;124:425-33.

42 Dworakowski $\mathrm{R}$, Wendler O. Optimal pain management after aortic valve implantation: an opportunity to improve outcomes after transapical access in the future? Heart 2012;98:1541-2.

43 Stortecky S, Buellesfeld L, Wenaweser P, et al. Transcatheter aortic valve implantation: the procedure. Heart 2012;98:iv44-51.

44 Amat-Santos IJ, Dumont E, Villeneuve J, et al. Effect of thoracic epidural analgesia on clinical outcomes following transapical transcatheter aortic valve implantation. Heart 2012;98:1583-90.

45 Bourantas CV, Farooq V, Onuma Y, et al. Transcatheter aortic valve implantation: new developments and upcoming clinical trials. Eurolntervention 2012;8:617-27.

46 Mussardo M, Latib A, Chieffo A, et al. Periprocedural and shortterm outcomes of transfemoral transcatheter aortic valve implantation with the Sapien XT as compared with the Edwards Sapien valve. JACC Cardiovasc Interv 2011;4:743-50.

47 Svensson LG, Tuzcu M, Kapadia S, et al. A comprehensive review of the PARTNER trial. J Thorac Cardiovasc Surg 2013;145:S11-16.

48 Hahn RT, Pibarot P, Stewart WJ, et al. Comparison of transcatheter and surgical aortic valve replacement in severe aortic stenosis: a longitudinal study of echo parameters in cohort A of the PARTNER trial. J Am Coll Cardiol 2013;61:2514-21.

49 Kempny A, Diller G-P, Kaleschke G, et al. Impact of transcatheter aortic valve implantation or surgical aortic valve replacement on right ventricular function. Heart 2012;98:1299-304.

50 Mesa D, Castillo F, Ruiz Ortiz M, et al. Impact of transcatheter aortic valve implantation or surgical aortic valve replacement on right ventricular function. Heart 2013;99:286.

51 Dworakowski R, Wendler O, Bhan A, et al. Successful transcatheter aortic valve implantation (TAVI) is associated with transient left ventricular dysfunction. Heart 2012;98:1641-6. 
52 Abdel-Wahab M, Zahn R, Horack M, et al. Aortic regurgitation after transcatheter aortic valve implantation: incidence and early outcome. Results from the German transcatheter aortic valve interventions registry. Heart 2011;97:899-906.

53 Gripari P, Ewe SH, Fusini L, et al. Intraoperative 2D and 3D transoesophageal echocardiographic predictors of aortic regurgitation after transcatheter aortic valve implantation. Heart 2012;98:1229-36.

54 Neragi-Miandoab S, Skripochnik E, Michler RE. Recently patented and widely used valves for transcatheter aortic valve implantation. Recent Pat Cardiovasc Drug Discov 2012;7:196-205.

55 Piazza N, Grube E, Gerckens U, et al. Procedural and 30-day outcomes following transcatheter aortic valve implantation using the third generation (18 Fr) corevalve revalving system: results from the multicentre, expanded evaluation registry 1-year following CE mark approval. Eurolntervention 2008;4:242-9.

56 Tamburino C, Capodanno D, Ramondo A, et al. Incidence and predictors of early and late mortality after transcatheter aortic valve implantation in 663 patients with severe aortic stenosis. Circulation 2011;123:299-308.

57 Bagur R, Manazzoni JM, Dumont E, et al. Permanent pacemaker implantation following isolated aortic valve replacement in a large cohort of elderly patients with severe aortic stenosis. Heart 2011;97:1687-94.

58 Fairbairn TA, Mather AN, Bijsterveld P, et al. Diffusion-weighted MRI determined cerebral embolic infarction following transcatheter aortic valve implantation: assessment of predictive risk factors and the relationship to subsequent health status. Heart 2012;98:18-23.

59 Prendergast BD, Naber CK, Popma JJ. Transatlantic perspectives on TAVI: from essential infrastructure and integration to expansion, research and development. Heart 2012;98:iv37-43.

60 Astarci P, Glineur D, Kefer J, et al. Magnetic resonance imaging evaluation of cerebral embolization during percutaneous aortic valve implantation: comparison of transfemoral and trans-apical approaches using Edwards Sapiens valve. Eur J Cardiothorac Surg 2011;40:475-9.

61 Naber CK, Ghanem A, Abizaid AA, et al. First-in-man use of a novel embolic protection device for patients undergoing transcatheter aortic valve implantation. Eurolntervention 2012;8:43-50.
62 Onsea K, Agostoni P, Samim M, et al. First-in-man experience with a new embolic deflection device in transcatheter aortic valve interventions. Eurolntervention 2012;8:51-6.

63 Nietlispach F, Wijesinghe N, Gurvitch R, et al. An embolic deflection device for aortic valve interventions. JACC CardiovasC Interv 2010;3:1133-8.

64 Krum H, Schlaich M, Whitbourn R, et al. Catheter-based renal sympathetic denervation for resistant hypertension: a multicentre safety and proof-of-principle cohort study. Lancet 2009;373:1275-81.

65 Investigators SH-. Catheter-based renal sympathetic denervation for resistant hypertension: durability of blood pressure reduction out to 24 months. Hypertension 2011;57:911-17.

66 Esler MD, Krum H, Sobotka PA, et al. Renal sympathetic denervation in patients with treatment-resistant hypertension (the Symplicity HTN-2 trial): a randomised controlled trial. Lancet 2010;376:1903-9.

67 Esler MD, Krum H, Schlaich M, et al. Renal sympathetic denervation for treatment of drug-resistant hypertension: one-year results from the Symplicity HTN-2 randomized, controlled trial. Circulation 2012;126:2976-82.

68 Hering D, Mahfoud F, Walton AS, et al. Renal denervation in moderate to severe CKD. J Am Soc Nephrol 2012;23:1250-7.

69 Schofer J. REDUCE-HTN trial. EuroPCR. Paris, France: Euro PCR 2013.

70 Worthley SG. EnligHTN-1: BP drops durable, safe at one year, as renal-denervation mania grows. EuroPCR. Paris, France: heartwire, 2013.

71 Templin C, Jaguszewski M, Ghadri JR, et al. Vascular lesions induced by renal nerve ablation as assessed by optical coherence tomography: pre- and post-procedural comparison with the Simplicity catheter system and the Enlig HTN ${ }^{\mathrm{TM}}$ multi-electrode renal denervation catheter. Eur Heart J 2013 Apr 25. [Epub ahead of print] doi:10.1093/eurheartj/eht141

72 Ewen S, Ukena C, Böhm M, et al. Percutaneous renal denervation: new treatment option for resistant hypertension and more? Heart 2013.

73 Meier P, Timmis A. Almanac 2012: interventional cardiology: the national society journals present selected research that has driven recent advances in clinical cardiology. Heart 2012;98:1701-9. 